\title{
Targeted Adversarial Learning Optimized Sampling
}

\author{
Jun Zhang ${ }^{1}$, Yi Isaac Yang ${ }^{2,3}$, and Frank Noé ${ }^{1, *}$
}

1 Department of Mathematics and Computer Science, Freie Universität Berlin, Arnimallee 6, 14195 Berlin, Germany

2 Department of Chemistry and Applied Biosciences, ETH Zurich, c/o USI Campus, Via Giuseppe Buffi 13, CH-6900, Lugano, Ticino, Switzerland

3 Institute of Computational Science, Università della Svizzera italiana (USI), Via Giuseppe Buffi 13, CH6900, Lugano, Ticino, Switzerland

* To whom correspondence should be addressed: (F.N.) frank.noe@fu-berlin.de

\begin{abstract}
Boosting transitions of rare events is critical to modern-day simulations of complex dynamic systems. We present a novel approach to modify the potential energy surface in order to drive the system to a user-defined target distribution where the free energy barrier is lowered. The new approach, called targeted adversarial learning optimized sampling (TALOS), cross-fertilizes statistical mechanics and deep learning. By casting the enhanced sampling problem as a competing game between a real sampling engine and a virtual discriminator, TALOS enables unsupervised construction of bias potential on an arbitrary dimensional space and seeks for an optimal transport plan that transforms the system into target. Through multiple experiments we show that on-the-fly training of TALOS benefits from the state-of-art optimization techniques in deep learning, thus is efficient, robust and interpretable. TALOS can also simultaneously learn to extract good reaction coordinate from a high-dimensional space where bias potential is being constructed. Additionally, TALOS is shown to be closely related to reinforcement learning, giving rise to a new framework of manipulating Hamiltonian in order to fulfill user-specified tasks via deep learning.
\end{abstract}

Key words: Deep learning, Rare events, Reinforcement learning, Optimal transport.

\section{Introduction}

Modern-day atomistic simulations have aided scientists to gain insights into some important chemical and biological processes. Despite of the progress, many dynamic events of interest, e.g. protein folding and ligand bindings, are embedded in a complex free-energy landscape where long-lived metastable states are separated by kinetic bottlenecks (i.e. free-energy barriers) which impede the transitions[1]. Therefore, timescales of these rare events are well out of the reach of brute-force simulations. Special-purpose hardware can partially ameliorate this issue but is still far from solving it[2]. To this end, much endeavour has been involved in development of enhanced sampling methods $[3,4,5,6]$ that allow investigating long-timescale properties of complex systems in an affordable computational time, overcoming kinetic bottlenecks, and exploring different metastable states.

Among the plethora of work on this topic, a large class of enhanced sampling methods aim at promoting the fluctuations of the slowly-changing collective variables (CVs) so as to favour transitions from one metastable state to another, including but not limited to umbrella sampling[7], metadynamics[8] and variationally enhanced sampling (VES)[9], and they differ with each other in how to choose CVs and how to construct the bias potential. Although a burst of recent work introduces machine learning to revolutionize the way of finding $\mathrm{CVs}[10,11]$, the way to build bias potential has hardly benefited from machine learning community yet. The reason behind is that, it is non-trivial to define an optimizable target within the framework of most existing enhanced sampling methods in order to allow the hybridization with deep learning.

As a prominent exception, VES first introduces a target distribution over the chosen CVs, then optimizes a bias potential based on them to minimize the Kullback-Leibler (KL) divergence between samples from the biased simulation and the target distribution[9]. However, since KL-divergence is not a continuous measure of the difference between two distributions[12], VES suffers from gradient vanishing or exploding issues when 
the distributions seldom overlap. Besides, VES works only if the target distribution and bias potential are defined in the same CV space and lacks of flexibility. In this paper, we introduce TALOS as a novel solution to a more general problem: unsupervised manipulation of Hamiltonian in order to drive the system towards a user-defined target distribution without any above-mentioned limitations. TALOS hybridizes statistical mechanics with state-of-art deep unsupervised learning methods, and is closely connected to reinforcement learning. Therefore, it is applicable to a variety of applications in physics where inference or modification to the Hamiltonian is needed in order to fulfill user-specified tasks.

\section{Methods}

We first define a target distribution (see more details about target distribution in SI) over a space of descriptors (or order parameters), based on which we can draw target (e.g. via Monte Carlo) and calculate ensemble averages. We now formulate the enhanced sampling problem as a minimax game (similar to the key idea of generative adversarial learning proposed by Goodfellow et al.[13]) between a virtual discriminator $\left(D_{w}\right)$ which maximally tells the difference between the target samples and produced ones, against a real sampler (e.g. MD engine) with a bias potential added to the Hamiltonian which aims to fool the virtual discriminator with produced samples (Fig. 1). The training ends when the Nash equilibrium of the game is reached.

Put it mathematically, given the Hamiltonian of the system $H(\mathbf{R})$ as a function of coordinates $\mathbf{R}$, we can design a bias potential $V_{\theta}(\mathbf{R})$, with $\theta$ denoting the trainable parameters (see SI for more information about functional forms $\left.V_{\theta}(\mathbf{R})\right)$. Let $\mathbf{q}(\mathbf{R})$ denote a space spanned by descriptors, based on which we define the target distribution $\mathbb{P}_{\text {target }}=p(\mathbf{q})$, and draw $\mathrm{MC}$ samples accordingly. Note that $\mathbf{q}(\mathbf{R})$ is only required to be a evaluable mapping from $\mathbf{R}$ to $\mathbf{q}$, but not necessarily differentiable. On the other hand, $\mathbb{P}_{\theta}$ denotes the distribution over q produced by the MD sampler running over $H(\mathbf{R})+V_{\theta}(\mathbf{R})$. The entire training process of TALOS is equivalent to solve the following minimax problem:

$$
\min _{V_{\theta}(\mathbf{R})} \max _{D_{w} \sim \mathbb{L} 1} \mathbf{W}\left(\mathbb{P}_{\text {target }}, \mathbb{P}_{\theta}\right)=\min _{V_{\theta}(\mathbf{R})} \max _{D_{w} \sim \mathbb{L} 1}\left\{\mathbb{E}_{\mathbf{q} \sim \mathbb{P}_{\text {target }}}\left[D_{w}(\mathbf{q})\right]-\mathbb{E}_{\mathbf{q} \sim \mathbb{P}_{\theta}}\left[D_{w}(\mathbf{q})\right]\right\}
$$

where $\mathbf{W}\left(\mathbb{P}_{\text {target }}, \mathbb{P}_{\theta}\right)$ is the Earth-Mover (EM) or Wasserstein-1 (W1) distance[14] (see SI for more information) telling the difference between the target and biased distributions; $D_{w}$ (q) belongs to the family of 1-Lipschitz $(\mathbb{L} 1)$ functions mapping the descriptor space $\mathbf{q}$ to the real space $\mathbb{R}$. EM distance can be interpreted intuitively as the "cost" of the optimal transport plan that transforms $\mathbb{P}_{\theta}$ into $\mathbb{P}_{\text {target }}$ by moving "mass" from $\mathbb{P}_{\theta}$ to $\mathbb{P}_{\text {target }}$. Note that unlike KL-divergence, EM distance is a continuous measure of divergence between two distributions (see SI for more information), and giving good gradient almost everywhere (especially when the two distributions seldomly overlap)[12]. However, the analytical form of $D_{w}$ is unknown, so we use a neural network, parametrized by $w$, to approximate it by minimizing the following loss function:

$$
\mathscr{L}(w)=\left\langle D_{w}(\mathbf{q}(\mathbf{R}))\right\rangle_{V_{\theta}(\mathbf{R})}-\left\langle D_{w}(\mathbf{q})\right\rangle_{p(\mathbf{q})}+R(w)
$$

where $R(w)$ is a restraint for Lipschitz continuity[15]. Since the solution to Eq. 2 is arbitrary up to an additive constant, we also include a zero-level restraint in $R(w)$ so that $\left\langle D_{w}(\mathbf{q}(\mathbf{R}))\right\rangle_{V_{\theta}(\mathbf{R})} \approx 0$ (see SI for available forms of $R(w)$ ). On the other hand, once the discriminator is fixed, we can learn a bias potential $V_{\theta}(\mathbf{R})$ that reduces the EM distance by minimizing the following loss function (see SI for the derivation of Eq. 3):

$$
\begin{aligned}
\mathscr{L}(\theta) & =\left\langle\left(D_{w}(\mathbf{q}(\mathbf{R}))-\left\langle D_{w}(\mathbf{q}(\mathbf{R}))\right\rangle_{V_{\theta}(\mathbf{R})}\right) \beta V_{\theta}(\mathbf{R})\right\rangle_{V_{\theta}(\mathbf{R})} \\
& \approx\left\langle D_{w}(\mathbf{q}(\mathbf{R})) \beta V_{\theta}(\mathbf{R})\right\rangle_{V_{\theta}(\mathbf{R})}
\end{aligned}
$$

where $\beta$ is the inverse temperature. We note here that Eqs. 2 and 3 bear intriguing similarity with the ActorCritic (AC) algorithm[16], hence rendering TALOS an alternative interpretation in terms of reinforcement learning: Eq. 2 is parallel to the training of a critic which is used to approximate the value function of being in a state $\mathbf{q}(\mathbf{R})$; whereas Eq. 3 is analogous to the policy gradient algorithm[17] for an actor who visits state $\mathbf{q}(\mathbf{R})$ with a probability proportional to $\exp \left(-\beta\left(H(\mathbf{R})+V_{\theta}(\mathbf{R})\right)\right)$ in order to maximize the expected value $\left\langle D_{w}(\mathbf{q}(\mathbf{R}))\right\rangle_{V_{\theta}(\mathbf{R})}$ (see SI for more discussion). $\mathscr{L}(w)$ indicates how close $\mathbb{P}_{\theta}$ is to $\mathbb{P}_{\text {target }}$ whereas $\mathscr{L}(\theta)$ measures the negative of the expected reward $\left(D_{w}(\mathbf{q}(\mathbf{R}))\right)$, thus both can be employed to monitor the training process. With this insight, one can develop variants of TALOS by defining other task-specific value functions and manipulate the Hamiltonian accordingly. 
In order to fulfill a robust on-the-fly training, we exploit three techniques arising from deep learning: Mini-batch optimization[18], stochastic gradient decent (SGD)[19] and data augmentation[20], respectively. For TALOS, a mini-batch of data is collected by running a short MD trajectory and recording uncorrelated samples (Fig. 1), based on which we update parameters of the critic network and bias potential. To reduce the fluctuations in the estimated expectation value in Eq. (3) due to mini-batch sampling, it is necessary to perform parameter update with stochastic optimization methods such as Adam[21] (see SI for more information). Besides, it is useful to further reduce the fluctuations through data augmentation. Two approaches can be adopted to this end: 1) multi-agent exploration through parallel sampling as in multiple-walkers metadynamics[22], and 2) experience replay[23, 24] to make full use of deposited samples[25] (see SI for more information) hence reducing the cost for generating data. To guarantee the Nash equilibrium reachable, we adopt two-timescale updated rule (TTUR)[26]: to train $D_{w}$ with a higher rate than $V_{\theta}$; or equivalently, to train multiple rounds for the discriminator first, followed by one round for the bias potential, and repeat this alternating procedure (see Algorithm S1).

\section{Results}

We first tested TALOS on the Berezhkovskii-Szabo (BS) potential[27] which consists of two local minima separated by an energy barrier (Fig. 2a), for proof of concept and benchmark of performance. It has been revealed[27, 28] that $q^{*}=x \cos \phi+y \sin \phi$ (with the skewed angle $\phi=32^{\circ}$ ) is an optimal linear reaction coordinate (Fig. 2a) to characterize the transitions between the two local minima, so we can directly employ $q^{*}$ as a $1 \mathrm{D}$ descriptor to define the target distribution and premise the bias potential. The target distribution follows a well-tempered (WT) form (Fig. 2b, see SI for more details). For bias potential, Legendre polynomials were chosen as basis functions (as in VES) whose expansion coefficients remained to be learned (Table S1), and the biased PMF can be numerically calculated once the expansion coefficients were obtained. We found that the loss function $\mathscr{L}(\theta)$ correlates well with the quality of the biased distribution hence can be used to check and control the training process of TALOS. The loss function reached a plateau after 30 to 40 iterations which serves as a stop sign for training, and the biased distribution indeed converged to the target one (Fig. 2c), demonstrating the feasibility and efficiency of our method. Besides, it is intriguing to find that the learning process of TALOS is highly interpretable in that TALOS dynamically updates the bias potential in a manner similar to metadynamics, although global basis functions are used instead of local Gaussian thus being more robust. The discriminator appears to be able to "feel" the difference between the target and current distributions so that the bias potential was changed to penalize where is over-sampled and encourage where is under-sampled (Figs. 2d and 2e).

The previous examples showed that TALOS works well when the target distribution and bias potential are based on the same space. However, in many real-world tasks, we may have some order parameters to distinguish different states, but have to enhance fluctuations of other degrees of freedom to boost the transitions; Or the target distribution is defined on a non-differentiable space, while we have to tune parameters on a differentiable space. In this respect, the ability of TALOS to construct bias potential on a space different from the one used to define the target distribution turns out to be very appealing, and we carried out two representative experiments over the same BS potential to test such property. In the first example (Case 1), we defined a WT target distribution over the 2D $(x, y)$ space but built bias potential only along the 1D $q^{*}$ defined as above. In the other example (Case 2), we defined a WT target distribution over the 1D $q^{*}$, but built bias potential on the 2D $(x, y)$ space instead. After training converged, we projected the original, target and biased distributions, respectively, onto $q^{*}$, and found that in both scenarios (Fig. S1a for Case 1 and S1b for Case 2), the learned bias potential drove the system to the desired distribution and flattened the PES (Fig. S1c for Case 1 and S1d for Case 2). However, noteworthy, the bias potentials in the two cases are quite different. The 1D bias potential (as commonly adopted in metadynamics and umbrella sampling) in Case 1 is rather crude (Fig. S1e), whereas in Case 2 the learned bias potential seems more reasonable and complementary to the original PES (Fig. S1f).

The interpretable learning process of TALOS to build high-dimensional bias potential given a target of different dimensions was also verified in an all-atom simulation of a $\mathrm{S}_{\mathrm{N}} 2$ reaction of $\mathrm{Cl}^{-}+\mathrm{CH}_{3} \mathrm{Cl} \longleftrightarrow$ $\mathrm{CH}_{3} \mathrm{Cl}+\mathrm{Cl}^{-}$(Fig. 3a) where the difference between the two $\mathrm{C}-\mathrm{Cl}$ bond distances $q^{*}=d_{1}-d_{2}$ is used as 1D descriptor (Fig. 3b) to define the target distribution (Fig. 3c) while a $2 \mathrm{D}$ bias potential of $\left(d_{1}, d_{2}\right)$ is constructed (see SI for more details). During training, the bias potential was lifted to compensate the extra time spent in the local minima. As a result, the system was driven to the predefined target distribution giving rise to faster chemical transitions (Fig. 3d) with a bias potential complementary to the original FES 
(Fig. 3e) .

This observation leads to an intriguing question: How can we modify a PES elegantly in order to drive the system to a certain distribution, provided that in terms of mathematics, there are infinite ways to modify a high-dimensional PES into a desired low-dimensional distribution? Intuitively, it were ideal if we could find a way to impose minimal external work to drive the system into target distribution. This problem is closely related to the optimal transport and control theory[29, 30]. Noteworthy, Earth-Mover or Wasserstein1 distance adopted in TALOS is intrinsically a solution to such kind of optimal transport problem[14]. From the perspective of physics, given the fluctuation-dissipation theorem, this problem can be translated as, if we could find a dimension, the fluctuation of which correlates best with the transitions of the concerned rare events. To physicists, such dimension is well known as reaction coordinate. Therefore, by virtual of TALOS, it is possible to find good RCs by learning the most economic way to build bias potential in order to boost the rare events (see SI for more discussion).

We tested this idea on the BS model system (Fig. 2a). The target distribution and bias potential are premised on a putative 1D RC $\hat{q}(\phi)=\tanh (x \cos \phi+y \sin \phi)$, where the skewed angle $\phi$ was treated as a learnable parameter in addition to the expansion coefficients over $\hat{q}$. The training process of TALOS becomes self-adaptive: Given an initial angle $\phi_{0}$, we can define a target distribution, then use it to train the bias potential hence the skewed angle $\phi$ as well as $\hat{q}$ gets updated. In order to encourage exploitation of a prior knowledge, we add an auxiliary term to the loss function and find it helpful (Eq. S18, see SI for more details). During the training iteration, TALOS gradually learned an optimal linear projection of $(x, y)$ on to $\hat{q}$ with a skewed angle around 32 degrees (Fig. 4a), in good agreement with the reported optimal linear RC for this system. Meanwhile, the bias potential (defined by the skewed angle and expansion coefficients) successfully drove the system to the target distribution along $\hat{q}\left(\phi=32^{\circ}\right)$ (Fig. 4b). These encouraging results show the potential of TALOS to discover low-dimensional RC from a high-dimensional space on which the bias potential is being constructed.

Next we generalized TALOS to two more complex systems. The first one is a 2D 3-well potential (Fig. $\mathrm{S} 2 \mathrm{a})$ which leads to transitions on different timescales. We chose an optimal linear combination[28]] $q^{*}=$ $x \cos \phi+y \sin \phi$ with a skewed angle $\phi=81.5^{\circ}$ from $x$-axis as a 1D descriptor to define the well-tempered target distribution (Fig. S2b). The bias potential was constructed on the 2D $(x, y)$ space. Like previous examples, TALOS is able to drive the system to the target distribution (Fig. S2b) and spontaneously builds bias potential that complements to the original energy landscape (Fig. S2c), with an interpretable dynamic learning process (Fig. S2d): Although there is more than one timescale of transitions, TALOS still learns to penalize where has been over-sampled with positive bias potential, and encourage where is under-sampled with negative bias potential, and finally tweaks the system to the target distribution (Fig. S2b). In the second study, TALOS was applied to the all-atom MD simulation of alanine dipeptide in explicit water (Fig. $5 \mathrm{a})$ to boost the configurational transitions of $(\phi, \psi)$ torsional angles (Fig. 5b, see SI for more details). Two simulations were performed: in System A, TALOS was trained w.r.t. a 1D uniform target distribution of $\phi$; whereas in System B, TALOS was trained w.r.t. a 2D uniform target distribution of $(\phi, \psi)$. Both systems were boosted by a learned bias potential over $2 \mathrm{D}(\phi, \psi)$ space. The training process is remarkably efficient which leads to convergence less than 1 ns (Fig. S3), and finally flattens the $(\phi, \psi)$ FES (Figs. 5c and 5d). In line with previous observations, no matter a $1 \mathrm{D}$ or $2 \mathrm{D}$ target distribution is used, TALOS is able to construct bias potentials complementary to the 2D $(\phi, \psi)$-FES (Fig. 5e) and achieves the defined target distribution. Particularly noteworthy, in System A where the target is defined solely along $\phi$, the barrier along $\psi$ will manifest as slow diffusion terms that hinder the transitions of $\phi$. Therefore, TALOS manages to boost the transitions of $\phi$ not only through lowering the free energy barrier but also by enhancing the diffusion along it. Such performance sets remarkable boundaries between TALOS and other conventional CV-based sampling methods (e.g. umbrella sampling, metadynamics etc.), and indeed reminds one of the merits of generalized-ensemble-based methods (e.g. replica exchange methods, integrated tempering sampling etc).

\section{Concluding Remarks}

In this paper, we present TALOS as a new approach to boosting transitions of rare events. TALOS enables unsupervised modification to the PES in order to drive the system to a user-defined target distribution where the free energy barrier is lowered. Inspired by recent progress of deep unsupervised learning, TALOS casts the enhanced sampling problem as a competing game between a virtual discriminator and the sampling engine, and builds bias potential in order to reach the Nash equilibrium. Different from existing enhanced sampling methods, TALOS has an evaluable and interpretable optimization target which can be used to monitor and 
control the learning process. TALOS also benefits from state-of-the-art optimization techniques from deep learning community, thus being robust, efficient and computationally economic.

As one compelling feature, TALOS supports flexible choices of descriptors which are used to define the target distribution and the space used for building bias potential, so that one can enhance fluctuations of arbitrary degrees of freedom in order to achieve a certain user-defined distribution. This feature allows TALOS to boost rare events not only by reducing the free energy barrier but also by speeding up the diffusion along the chosen coordinates. Besides, since the loss function in TALOS approximates an optimal transport problem, TALOS may manage to drive the system to the target with minimal work. Such property renders TALOS potentiality to extract good low-dimensional RCs during the on-the-fly construction of bias potentials on a high dimensional space.

In addition to enhanced sampling, targeted adversarial learning can be a promising strategy for a wide range of applications. For instance, TALOS can be used to fit force field parameters with minimal adjustment that if we choose the target distribution to be that of the dihedral angles in the protein data bank, and regard the force field parameters of the MD engine as learnable parameters. Furthermore, since TALOS has a clear interpretation in terms of actor-critic reinforcement learning, one can simply devise other variants based on their own task-specific value functions. In this sense, the idea and theory behind TALOS open a door for physics community to embrace deep learning. Therefore, we expect the method, along with the theory and training protocol presented in this paper, to be appealing to a broad community in chemistry and physics.

Acknowledgments We thank Prof. Michele Parrinello, Prof. Yi-Qin Gao, GiovanniMaria Piccini and Haiyang Niu for useful discussion. Molecular dynamic simulations were carried out on the Euler Cluster at ETH Zürich. This research was supported by Alexander von Humboldt Foundation, and the VARMET European Union Grant ERC-2014-ADG-670227 and the NCCR MARVEL 51NF40_141828 funded by the Swiss National Science Foundation.

\section{References}

[1] Bryngelson, J. D.; Onuchic, J. N.; Socci, N. D.; Wolynes, P. G. Proteins: Struct., Funct., Bioinf. 1995, 21, 167-195.

[2] Lindorff-Larsen, K.; Piana, S.; Dror, R. O.; Shaw, D. E. Science 2011, 334, 517-520.

[3] Bernardi, R. C.; Melo, M. C.; Schulten, K. Biochimica et Biophysica Acta (BBA)-General Subjects 2015, $1850,872-877$.

[4] Bonomi, M.; Parrinello, M. Phys. Rev. Lett. 2010, 104, 190601.

[5] Okamoto, Y. J. Mol. Graphics Modell. 2004, 22, 425-439.

[6] Yang, L.; Liu, C.-W.; Shao, Q.; Zhang, J.; Gao, Y. Q. Acc. Chem. Res. 2015, 48, 947-955.

[7] Torrie, G. M.; Valleau, J. P. J. Comput. Phys. 1977, 23, 187-199.

[8] Barducci, A.; Bussi, G.; Parrinello, M. Phys. Rev. Lett. 2008, 100, 020603.

[9] Valsson, O.; Parrinello, M. Phys. Rev. Lett. 2014, 113, 090601.

[10] Wehmeyer, C.; Noé, F. J. Chem. Phys. 2018, 148, 241703.

[11] Mendels, D.; Piccini, G.; Parrinello, M. J. Phys. Chem. Lett. 2018, 9, 2776-2781.

[12] Arjovsky, M.; Chintala, S.; Bottou, L. arXiv preprint arXiv:1701.07875 2017,

[13] Goodfellow, I.; Pouget-Abadie, J.; Mirza, M.; Xu, B.; Warde-Farley, D.; Ozair, S.; Courville, A.; Bengio, Y. Generative adversarial nets. Advances in neural information processing systems. 2014; pp 26722680 .

[14] Andoni, A.; Indyk, P.; Krauthgamer, R. Earth mover distance over high-dimensional spaces. Proceedings of the nineteenth annual ACM-SIAM symposium on Discrete algorithms. 2008; pp 343-352. 
[15] Gulrajani, I.; Ahmed, F.; Arjovsky, M.; Dumoulin, V.; Courville, A. C. Improved training of wasserstein gans. Advances in Neural Information Processing Systems. 2017; pp 5767-5777.

[16] Mnih, V.; Badia, A. P.; Mirza, M.; Graves, A.; Lillicrap, T.; Harley, T.; Silver, D.; Kavukcuoglu, K. Asynchronous methods for deep reinforcement learning. International conference on machine learning. 2016; pp 1928-1937.

[17] Williams, R. J. Machine learning 1992, 8, 229-256.

[18] Ghadimi, S.; Lan, G.; Zhang, H. Math. Program. 2016, 155, 267-305.

[19] Bottou, L. Proceedings of COMPSTAT'2010; Springer, 2010; pp 177-186.

[20] Tanner, M. A.; Wong, W. H. J. Am. Stat. Assoc. 1987, 82, 528-540.

[21] Kingma, D. P.; Ba, J. arXiv preprint arXiv:1412.6980 2014,

[22] Raiteri, P.; Laio, A.; Gervasio, F. L.; Micheletti, C.; Parrinello, M. J. Phys. Chem. B 2006, 110, 3533-3539.

[23] Mnih, V. et al. Nature 2015, 518, 529.

[24] Ganin, Y.; Kulkarni, T.; Babuschkin, I.; Eslami, S.; Vinyals, O. arXiv preprint arXiv:1804.01118 2018,

[25] Bonomi, M.; Barducci, A.; Parrinello, M. J. Comput. Chem. 2009, 30, 1615-1621.

[26] Heusel, M.; Ramsauer, H.; Unterthiner, T.; Nessler, B.; Hochreiter, S. Gans trained by a two time-scale update rule converge to a local nash equilibrium. Advances in Neural Information Processing Systems. 2017; pp 6626-6637.

[27] Berezhkovskii, A.; Szabo, A. J. Chem. Phys. 2005, 122, 014503.

[28] Tiwary, P.; Berne, B. J. Chem. Phys. 2017, 147, 152701.

[29] Villani, C. Optimal transport: old and new; Springer Science \& Business Media, 2008; Vol. 338.

[30] Hartmann, C.; Schütte, C.; Weber, M.; Zhang, W. Probability Theory and Related Fields 2018, 170, 177-228. 


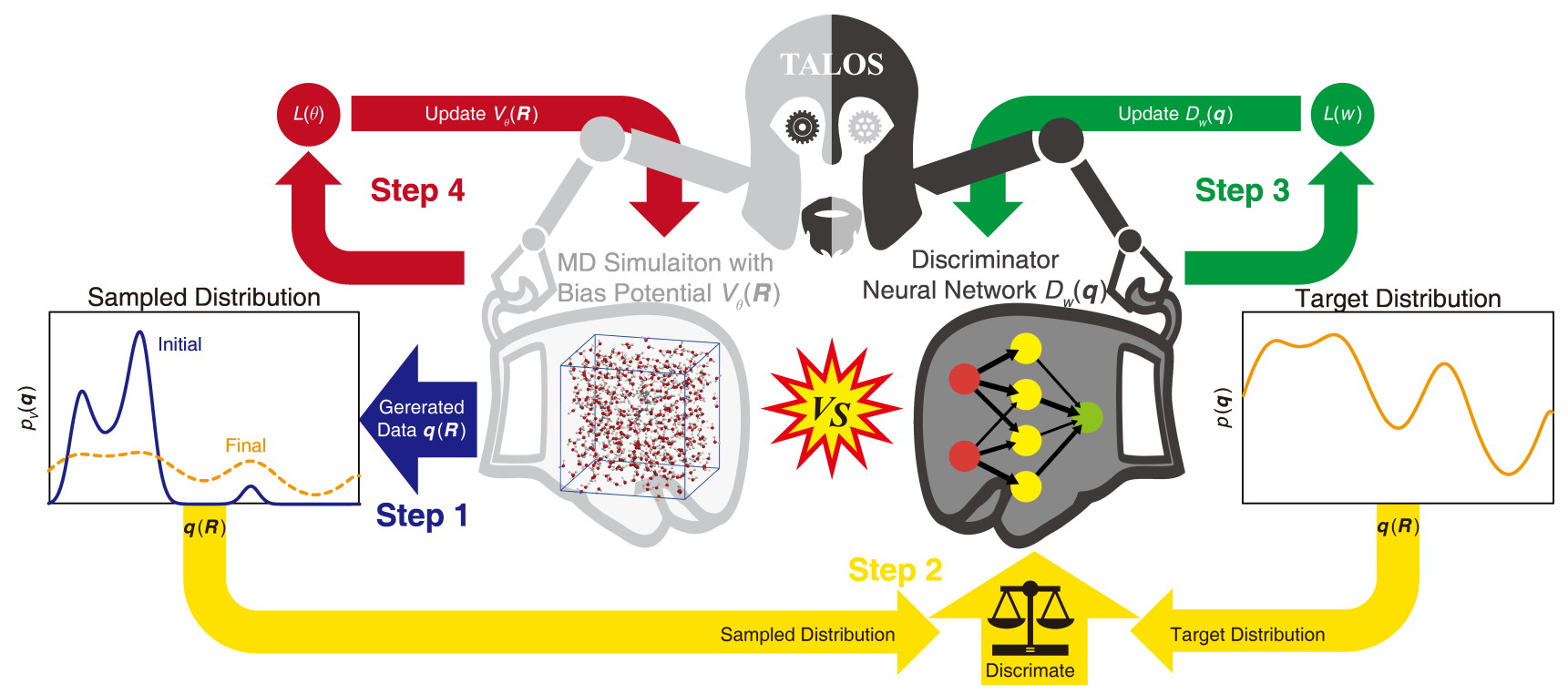

Figure 1: Illustration and workflow of targeted adversarial learning optimized enhanced sampling (TALOS). Target samples are drawn from a target distribution defined on a descriptor space (q), meanwhile a real sampler (MD engine) parallely generates samples based on the Hamiltonian modified by bias potential $V_{\theta}(\mathbf{R})$. A virtual discriminator (neural network) $D_{w}$ is trained to approximate the earth-mover distance between target and biased samples, and feedback to train the bias potential $V_{\theta}(\mathbf{R})$. 

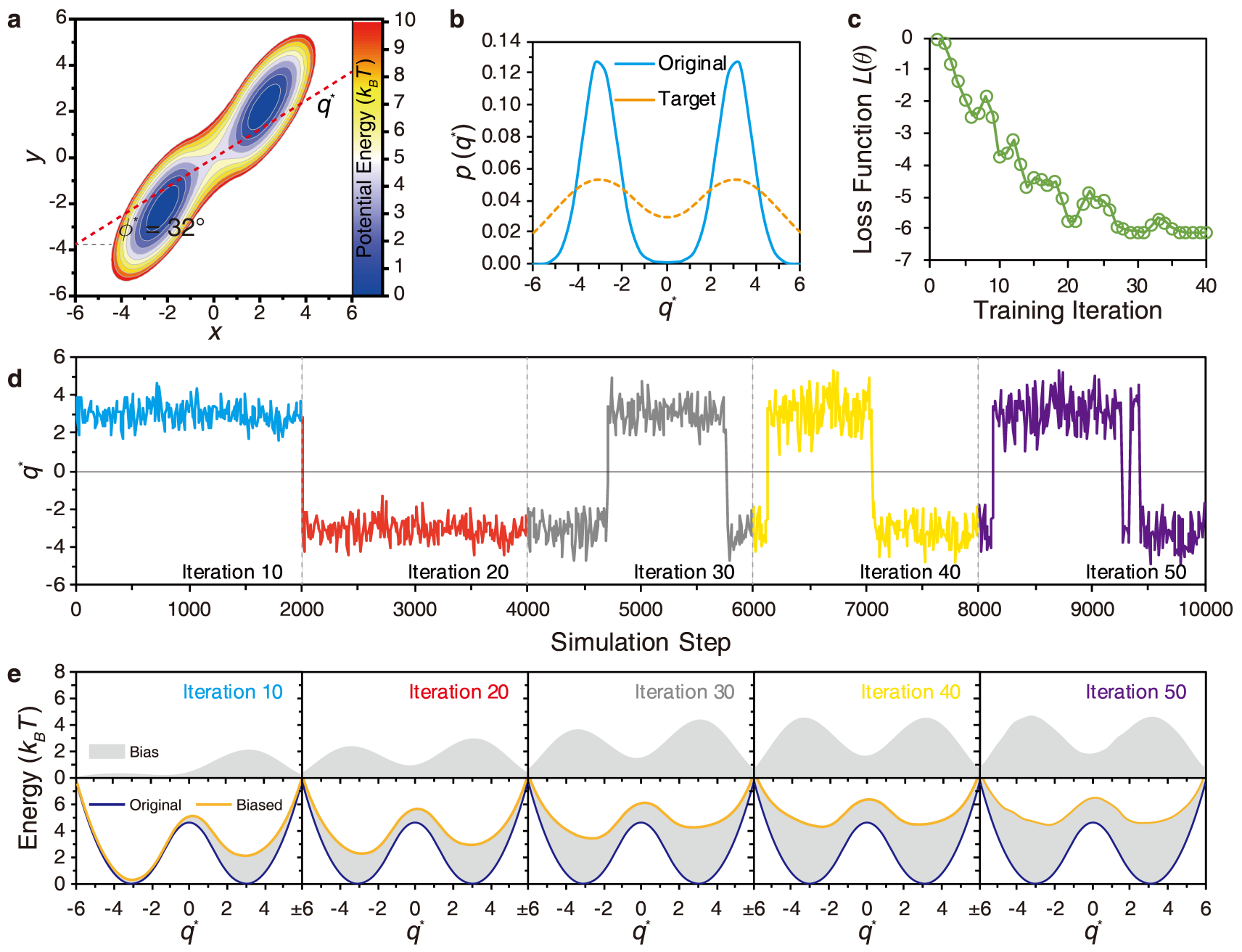

Figure 2: (a) 2D BS potential energy surface and the optimal linear $\mathrm{RC} q^{*}=x \cos \phi+y \sin \phi\left(\phi=32^{\circ}\right)$. (b) Original distribution (blue solid) and the target distribution (orange dotted) defined on $q^{*}$. (c) The loss function $\mathscr{L}(\theta)$ is plotted against training iterations. (d) Minibatch of biased MD samples (trajectories) projected on $q^{*}$ at different training iterations. (e) The learned bias potential (upper panels) and the effective biased PMF (orange lines in lower panels) at different training iterations. Original PMF (blue lines in lower panels) is plotted for comparison. 
a

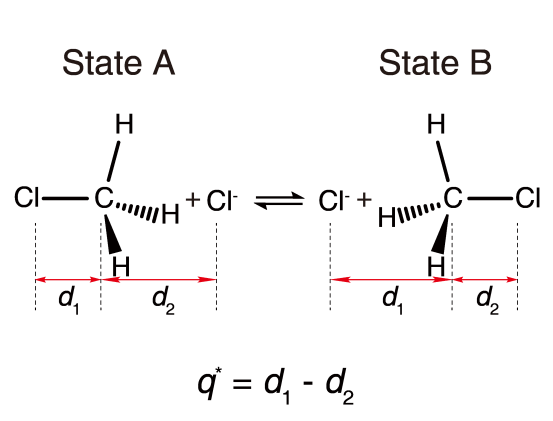

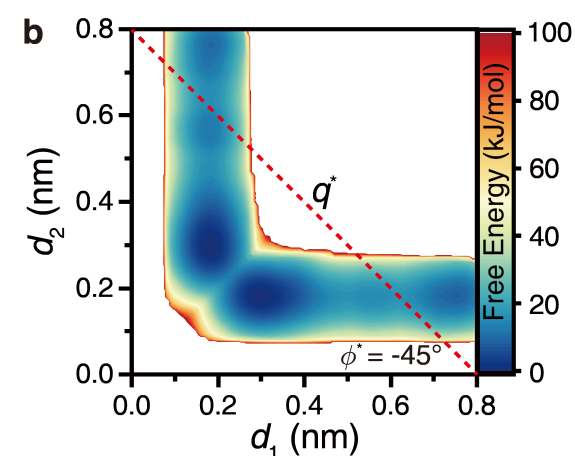

$100 \mathrm{C}$

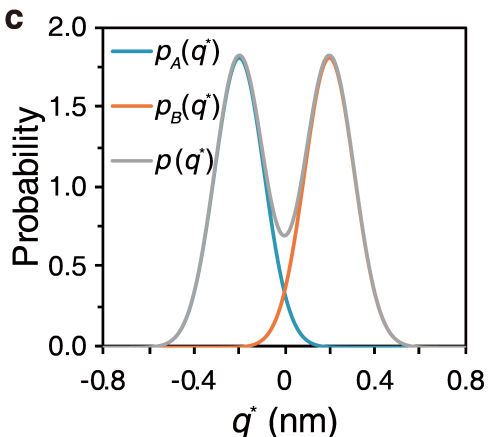

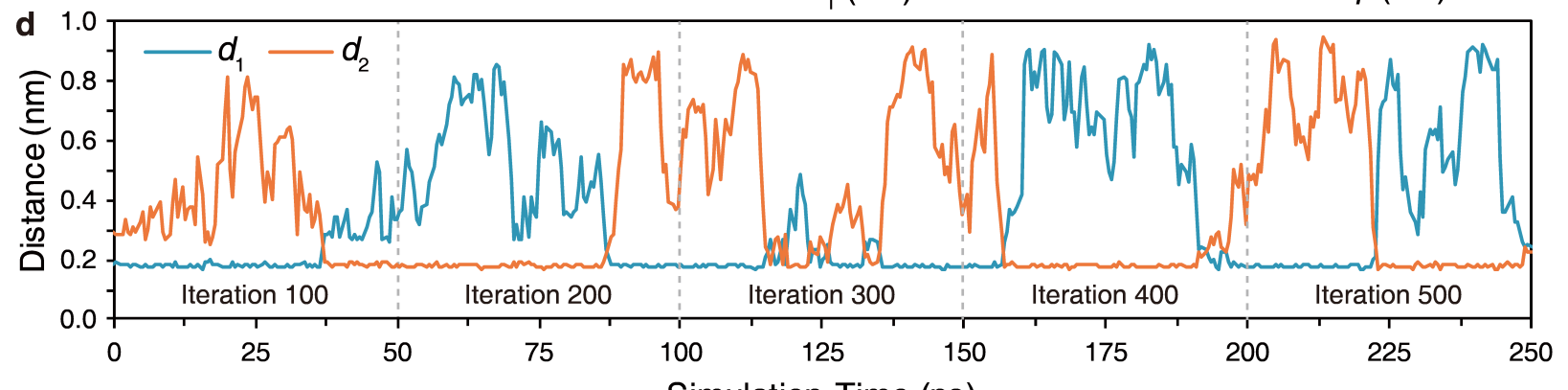

Simulation Time (ps)

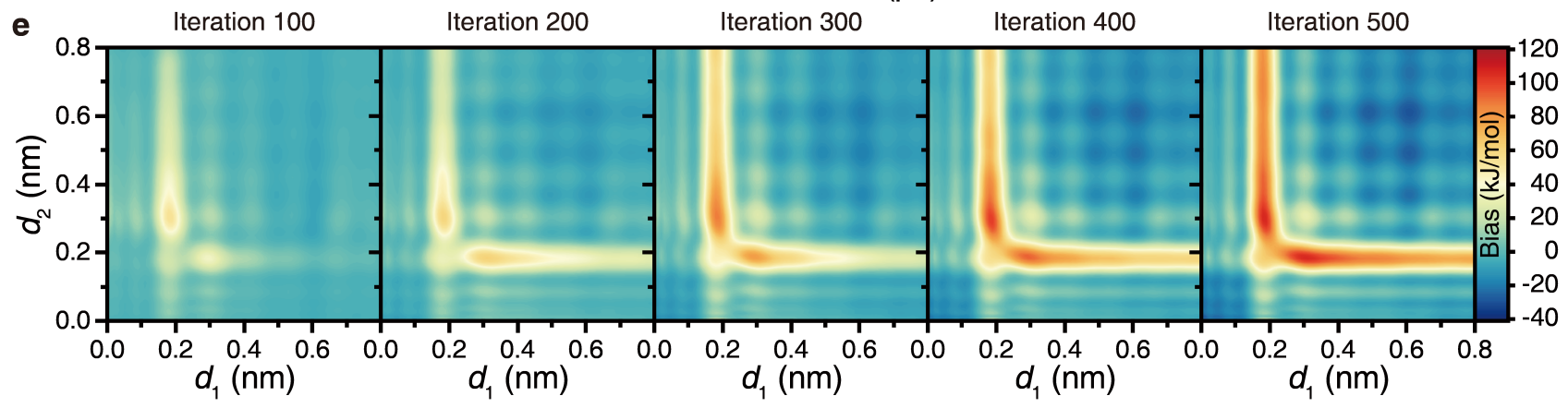

Figure 3: (a) Enhanced sampling of a symmetric $\mathrm{S}_{\mathrm{N}} 2$ reaction, $\mathrm{Cl}^{-}+\mathrm{CH}_{3} \mathrm{Cl} \longleftrightarrow \mathrm{CH}_{3} \mathrm{Cl}+\mathrm{Cl}^{-}$. The two $\mathrm{C}-\mathrm{Cl}$ bond distances $\left(d_{1}, d_{2}\right)$ are chosen as variables to build bias potential, and an optimal linear RC $q^{*}=d_{1}-d_{2}$ to define the target distribution. (b) The 2D PMF over $\left(d_{1}, d_{2}\right)$ space and the illustration of $q^{*}$. (c) The distribution of $q^{*}$ for the reactant (blue), product (orange) and the intrapolated target distribution (grey). (d) Minibatch of biased MD samples (trajectories) projected on $d_{1}$ (blue) and $d_{2}$ (orange) at different training iterations. (e) The learned 2D bias potential at different training iterations. 

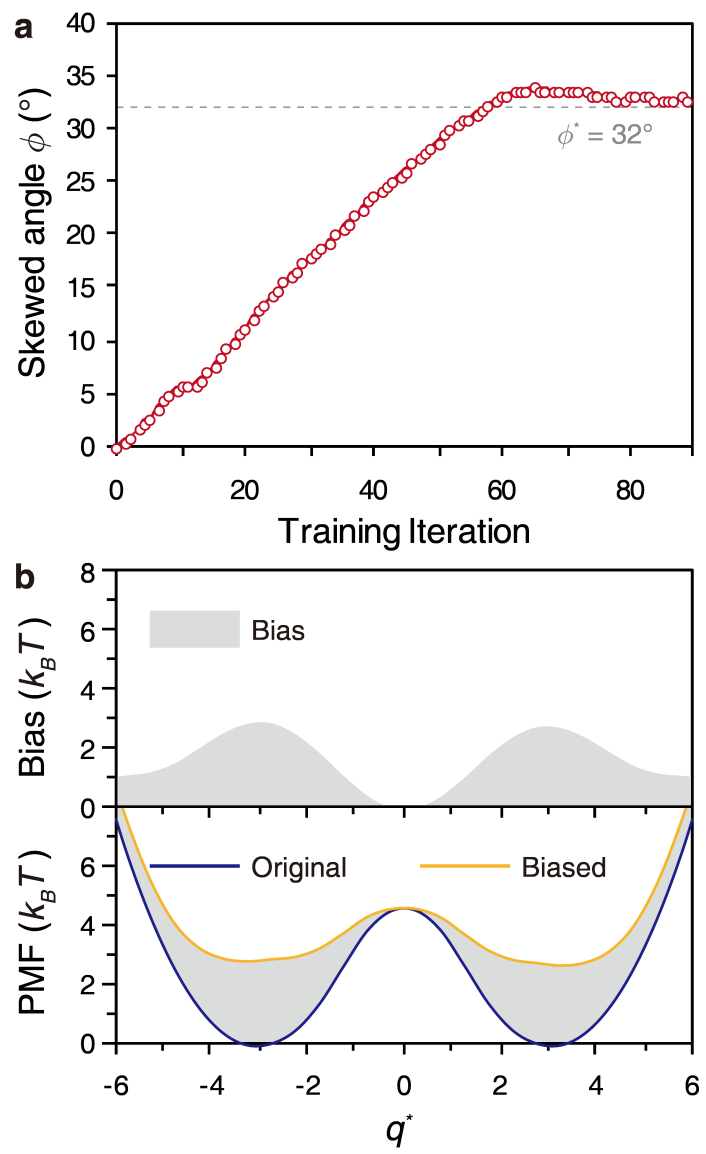

Figure 4: Adaptive learning of reaction coordinate. (a) Value of the skewed angle $\phi$ for the BS potential plotted against training iterations, where $q(\phi)=\tanh (x \cos \phi+y \sin \phi)$ is used to define the target and bias potential. Theoretical optimal value is shown in grey dashed line. (b) The converged 1D bias potential (upper panel) and biased PMF (orange line in lower panel) along the learned $q^{*}$ for the BS potential. Original PMF (blue line in lower panel) is plotted for comaprison. 
a<smiles>CNC(=O)CC(C)C(C)NC(C)=O</smiles>

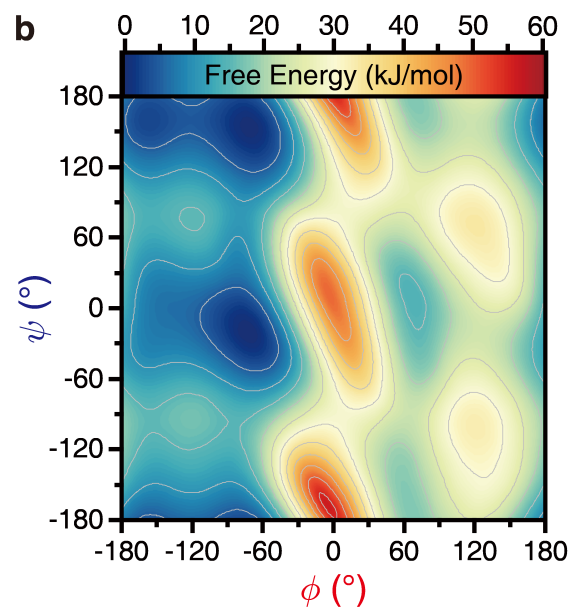

C
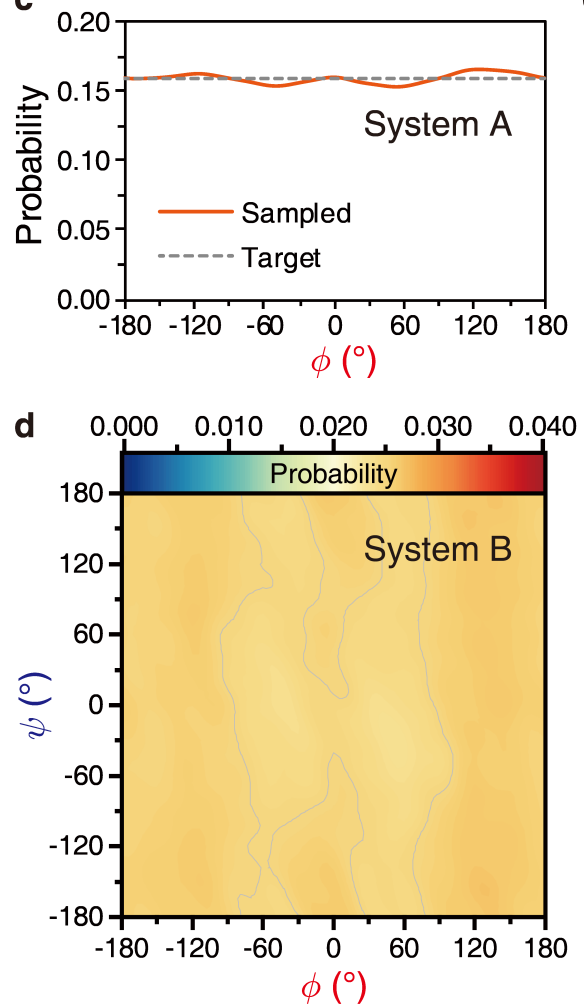

e
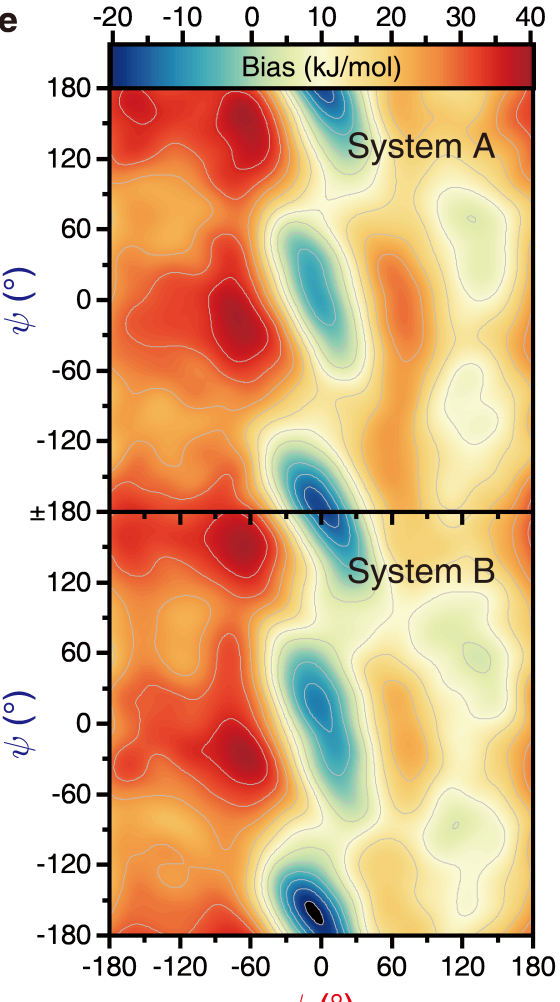

Figure 5: (a) Enhanced sampling of Ala2, where the torsional angles $(\phi, \psi)$ are slowly changing variables leading to configurational transitions, based on which we built bias potentials. (b) The original 2D PMF over $(\phi, \psi)$ space. (c) For system A, a uniform 1D target distribution is defined along $\phi$ (grey dashed), and the converged biased distribution projected is shown in red solid line. (d) For system B, the converged 2D biased distribution learned according to a uniform 2D target distribution defined over $(\phi, \psi)$. (e) The learned 2D bias potentials in System A (upper panel) and System B (lower panel), respectively. 